\title{
Titanium Proximal femoral Nailing for inter-trochanteric fractures of Femur
}

\author{
Jose Ashish K ${ }^{1}$, Surendher Kumar $\mathbf{R}^{2}$ \\ ${ }^{1}$ Dr Ashish Kumar Jose, ${ }^{2}$ Dr Surendher Kumar R, Department of Orthopaedics, Mahatma Gandhi Medical College \& \\ Research Institute, Pondicherry, India.
}

Address for correspondence: Dr Ashish Kumar Jose, Email: joseashish@yahoo.co.in

\begin{abstract}
Introduction: Inter-trochanteric fractures of femur are one of the commonest fractures encountered in orthopaedic practice. It is mostly seen in the older age groups. An increased incidence of these fractures is noted these days owing to the increased life expectancy. Several implants have been designed for the treatment of these fractures. In this study we analyze the functional and radiological outcome of inter-trochanteric fractures fixed with titanium proximal femoral nail. Methods: This is a prospective study. 23 patients with inter-trochanteric fractures treated by titanium proximal femoral nail from August 2012 to December 2014 were included in the study. We analyzed these 23 patients for functional and radiological out come and complications. Results: The fracture union rate was $100 \%$ and the average union time was 12 weeks. Varus mal-union was noted in 3 patients and shortening was seen in 1 patient. We did not encounter any infection in our study. 15 patients had excellent outcome, 7 had good outcome and 1 patient had fair outcome in our study (based on Kyle's criteria). Conclusion: The result of our study shows that titanium proximal femoral nail is an excellent implant for the fixation of inter-trochanteric fractures of femur with very good functional and radiological outcome.
\end{abstract}

Keywords: Inter-Trochanteric Fracture, Titanium Proximal Femoral Nail, PFN.

\section{Introduction}

An inter-trochanteric fracture of the femur occurs between the greater trochanter, which is the attachment of the gluteus medius and gluteus minimus, and the lesser trochanter which is the attachment of the iliopsoas muscle. Inter-trochanteric fractures of femur are one of the commonest fractures encountered by orthopaedic surgeons world over. The incidence of these fractures increases with advancing age. There has been a tremendous increase in the incidence of these fractures with the ever growing population, increased life expectancy and the increased incidences of motor vehicle accidents. These fractures commonly occur as a consequence of low energy falls in the elderly and rarely due to high energy trauma like road traffic accidents and fall from height. Earlier these fractures were managed by non surgical methods of prolonged bed rest, traction and immobilization. Despite giving acceptable results of fracture union the non surgical

Manuscript received: $1^{\text {st }}$ Sept 2015

Reviewed: $9^{\text {th }}$ Sept 2015

Author Corrected: $18^{\text {th }}$ Septt 2015

Accepted for Publication: $28^{\text {th }}$ Sept 2015 methods have become obsolete now due to the unacceptable morbidity and mortality associated with prolonged immobilization [1,2]. Surgical treatment of these fractures significantly reduces the morbidity and mortality by allowing early mobilization of the patient and thus avoiding the complications of prolonged bed rest [3]. Over the years, various implants have been designed for the treatment of inter-trochanteric fractures. The main objective of treatment is early mobilization with stable fixation using as minimal invasive procedure as possible.Owing to their biomechanical properties, intramedullary devices appear to be the ideal implant. There have been reports of intra and post operative complications in some cases fixed with intramedullary devices [4,5]. The proximal femoral nail was designed keeping in mind these complications. In this study we analyze the functional and radiological outcome of titanium proximal femoral nailing for inter-trochanteric fractures of the femur.

\section{Materials and Methods}


This is a prospective study. Patients with intertrochanteric fracture fixed using titanium proximal femoral nail between August 2012 and December 2014 were included in the study. Patients treated with stainless steel proximal femoral nail, other implants and those with other fractures in the same limb were not
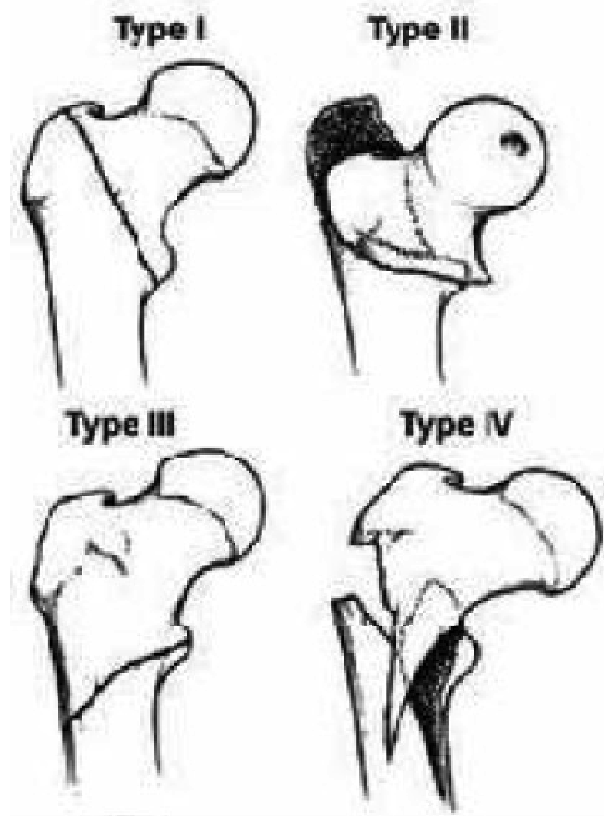

Fig.1: Boyd and Griffin classification included. During the study period, 23 patients of intertrochanteric fractures were treated with titanium proximal femoral nail. The minimum follow-up was 6 months. The fractures were classified based on Boyd and Griffin classification system (Fig.1,2) [6]:

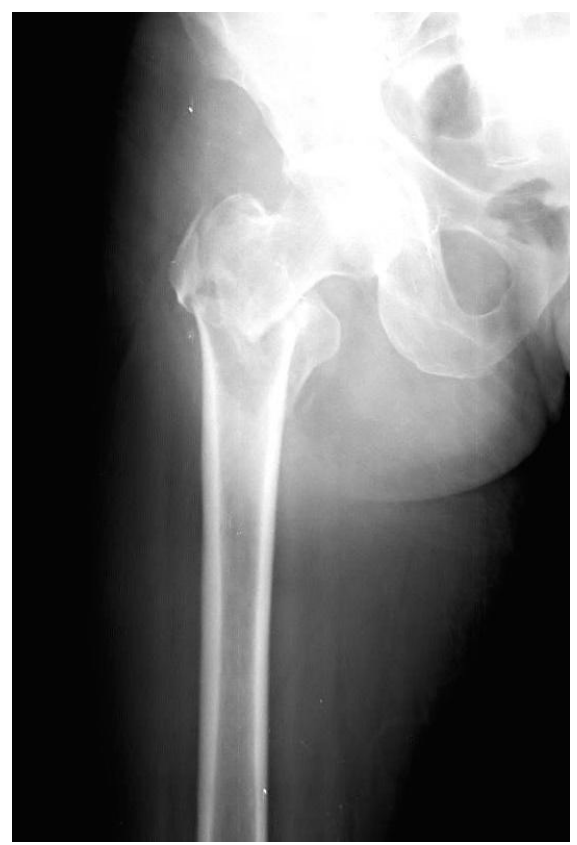

Fig 2: Inter-trochanteric fracture - Pre operative X-Ray

- Type I: Fractures that extend along the intertrochanteric line.

- Type II: Comminuted fractures with the main fracture along the intertrochanteric line but with multiple secondary fracture lines.

- Type III: Fractures that extend to or are distal to the lesser trochanter.

- Type IV: Fractures of the trochanteric region and proximal shaft with fractures in atleast two planes.

\section{Procedure (STEPS)}

- Patient placed supine on fracture table under spinal anesthesia.

- Affected leg was placed in a traction boot and the normal limb was flexed and abducted in lithotomy position to allow easy access for image intensifier (Fig.3).

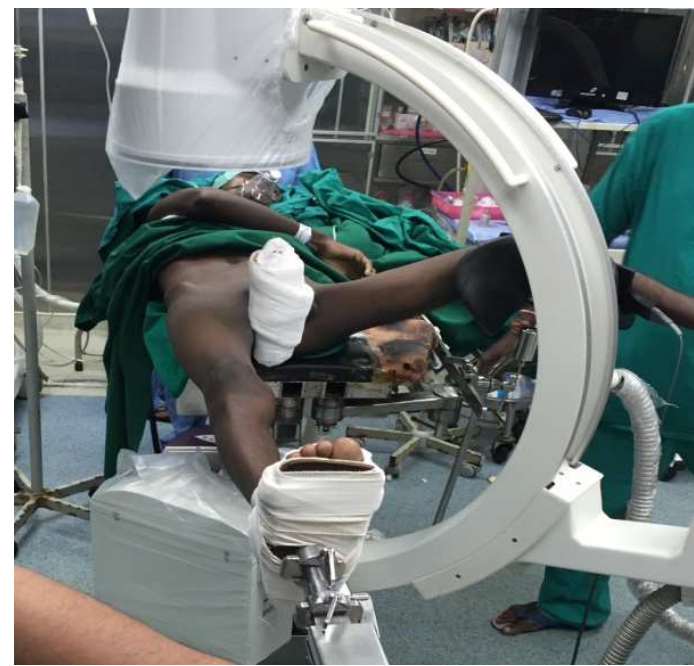

Fig 3: Patient positioning for surgery.

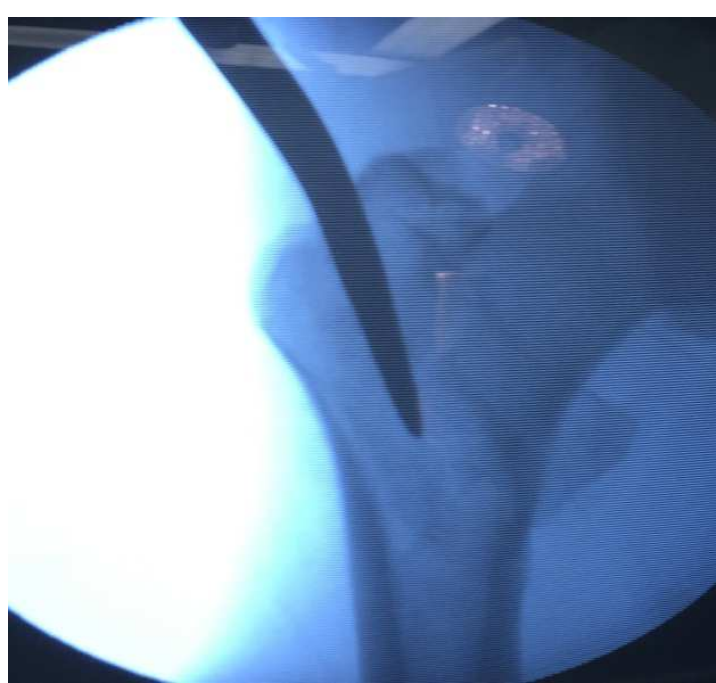

Fig 4 : Entry point made using bone awl 
- The fracture was reduced by traction and internal rotation.

- The reduction was checked both in antero-posterior and lateral views using the image intensifier.

- Parts painted and draped .

- $5 \mathrm{~cm}$ incision made, extending proximally from the greater trochanter.

- Entry point made at the tip of the greater trochanter using bone awl (Fig.4).

- Guide wire inserted and position checked under image intensifier (Fig.5).

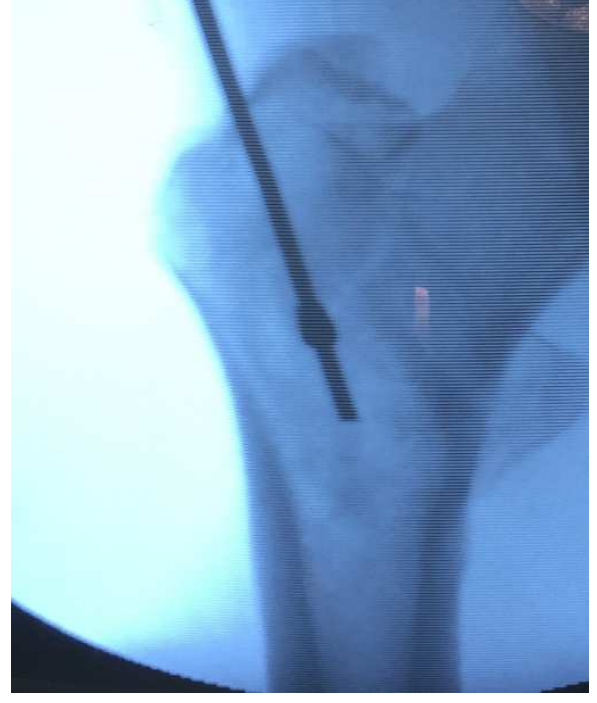

Fig 5: Insertion of the guide wire

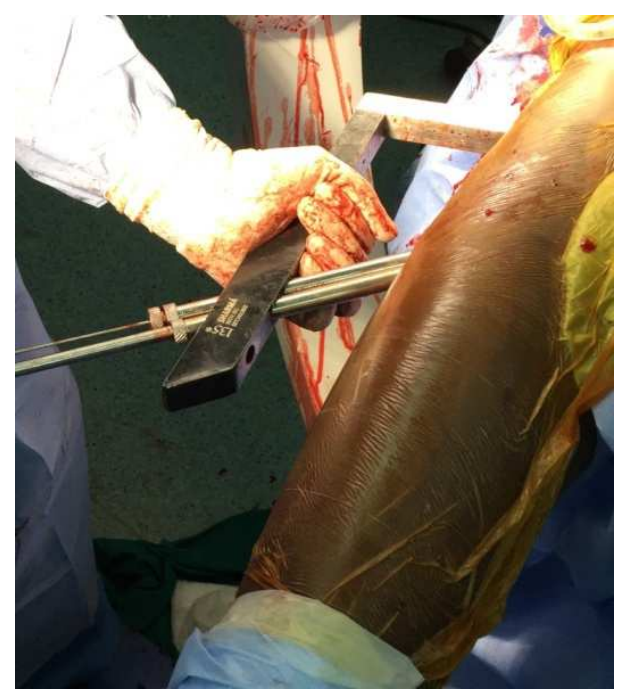

Fig 6: Insertion of the cervical lag screw

- Proximal reaming was done.

- The proximal femoral nail was inserted over the guide wire.

- Proximally the cervical lag screw and de-rotation screw are inserted with the help of the jig (Fig.6).

- Distal locking bolts are inserted using the jig (Fig.7).

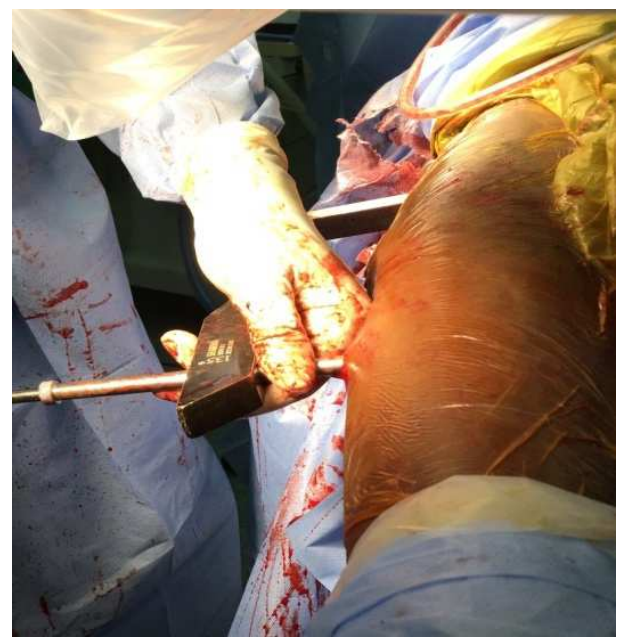

Fig 7: Distal locking

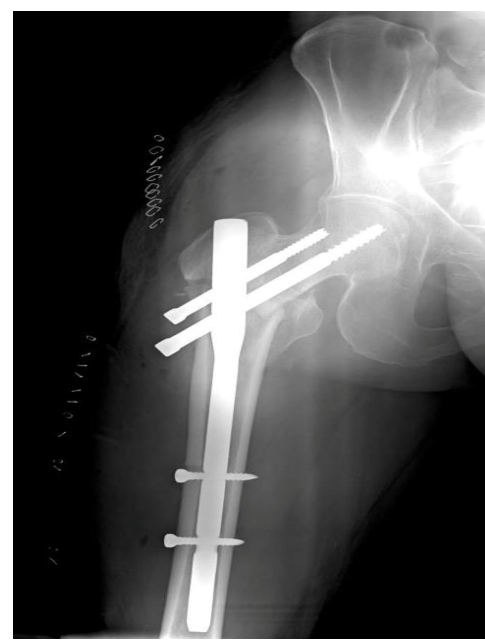

Fig 8: Immediate post operative X-Ray

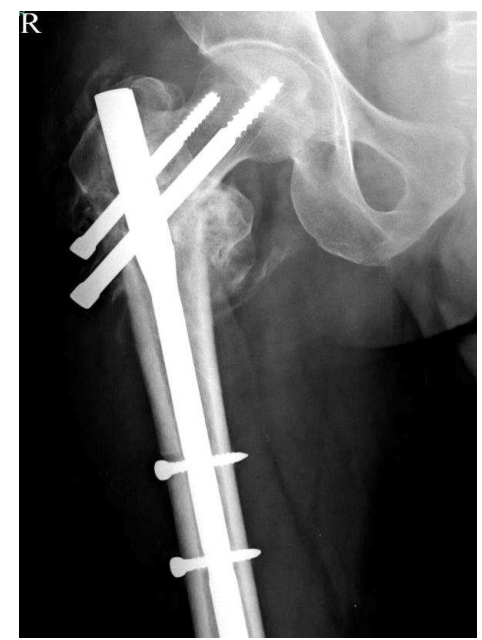

Fig 9: Fracture united at 12 weeks

- Wound closed in layers and sterile dressing applied.

- Check X-ray was taken immediate post-op. (Fig.8).

\section{Post Operative Protocol}

- $\quad$ Sit propped up and active quadriceps exercises on $1^{\text {st }}$ post operative day (POD).

- Stand with support on $2^{\text {nd }}$ POD. 
- Partial weight bearing walking with support from $3^{\text {rd }}$ POD.

- Suture removal on $12^{\text {th }}$ POD.

- Full weight bearing walking once there is radiological signs of union.

- Functional and radiological assessment at 6 weeks, 12 weeks and monthly until fracture union and every 6 months thereafter.

\section{Scoring System}

All patients were followed up until fracture union occurred. Kyle's criterion was used to evaluate the functional outcome [7]:

I . Excellent :

$>$ No or minimum limp

$>$ Absence of pain

$>$ Full range of motion

II. Good :

$>$ Mild limp

$>$ Mild occasional pain

$>$ Full range of motion

III. Fair :

$>$ Moderate limp

$>$ Moderate pain

$>$ Limited range of movement

IV. Poor :

$>$ Wheelchair bound

$>$ Pain on any position

Non-ambulatory

\section{Results}

Of the 23 patients, 15 were male and 8 were female with 11 patients injuring their right hip and 12 patients injuring their left hip. The age group varied from 39 years to 74 years with a mean age of 59 years. The mode of injury was trivial slip and fall in 19 patients and road traffic accidents in 4 patients.

The fractures were classified according to Boyd and Griffin classification [6]:

$>$ Type I : 1 patient

$>$ Type II : 13 patients

$>$ Type III : 9 patients

$>$ Type IV : Nil

Average operative duration was 50 minutes ( 45 - 75 minutes )

Average intra-operative blood loss : $300 \mathrm{ml}(200-330 \mathrm{ml})$

Duration of follow up: 6 months to 2 years

Mean follow up: 9 months

Union rate: $100 \%$

Union time: 10 weeks to 14 weeks (Average 12 weeks)

Varus mal-union: 3 patients

Shortening: 1 patient

Infection: Nil

Non union: Nil

Delayed union: Nil

Implant Failure: Nil

Based on the scoring system, the results of our study are:

$>$ Excellent : 15 patients 
$>$ Good : 7 patients

Fair : 1 patient

$>$ Poor : Nil

\section{Discussion}

Inter-trochanteric fractures are common in the elderly due to weak eyesight, poor muscle co-ordination, and associated co-morbidities like osteoporosis. Surgical treatment of these fractures significantly reduces the morbidity and mortality by allowing early mobilization, hence preventing the complications arising out of prolonged bed rest. Several implants have been designed for the treatment of these fractures. The use of Dynamic Hip Screw is quite popular but the increased incidence of uncontrolled collapse in comminuted fractures leading to varus mal-union has forced the surgeons to think of an intra-medullary option. In this study we assessed the functional and radiological outcome of inter-trochanteric fractures fixed with titanium proximal femoral nail.

The average operative duration in our study was 50 minutes with the longest duration being 75 minutes and the shortest being 45 minutes. This is similar to the study of Pajarinen et al [8], and significantly lower when compared to the study of Morihara (77 minutes) [9]. The average intra-operative blood loss in our study was $300 \mathrm{ml}$, which is almost similar to the study of Pajarinen et al (320ml) [8]. The average time for radiological union was 12 weeks. Varus mal-union was seen in 3 patients. Shortening was observed in 1 patient which was less than $2 \mathrm{~cm}$ and not significant. We did not encounter any infection, non-union, delayed union or implant failure in our study. Based on Kyle's criteria for functional outcome, 96\% (22 patients) of the patients has excellent to good results (Fig.10).

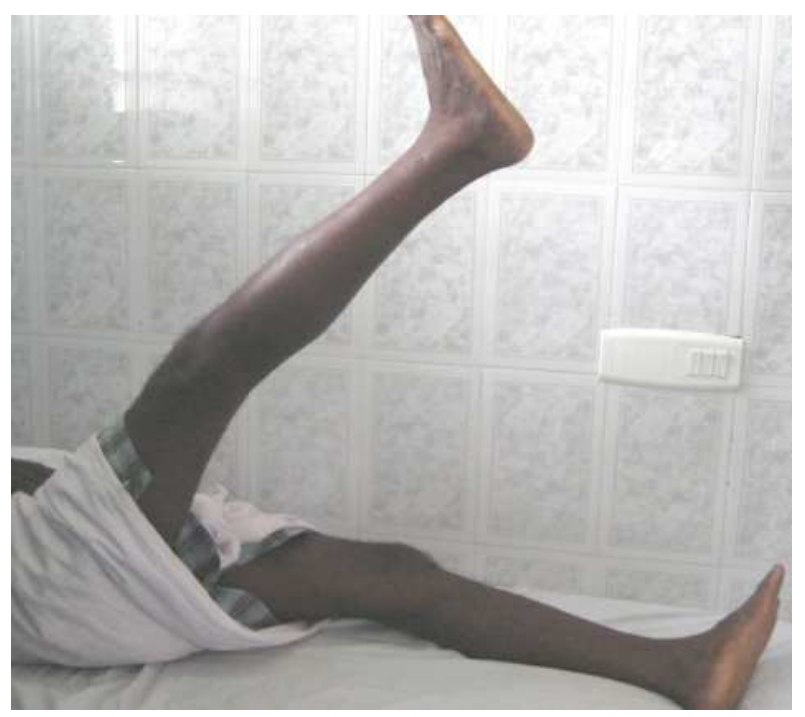

Fig.10: Good functional outcome

Titanium PFN (Proximal Femoral Nail) has several advantages over the DHS (Dynamic Hip Screw). The procedure for PFN is minimally invasive and being a load sharing device, the PFN has definite biomechanical advantages over the DHS. PFN prevents uncontrolled collapse and varus mal-union, which are the main complications of DHS fixation in comminuted inter-trochanteric fractures. PFN also provides high rotational stability to the head-neck fragment.

The aim of surgical fixation in these fractures should be to promote faster healing, early mobilization and return to pre-injury level of function as soon as possible.

\section{Conclusion}

The outcome of our study shows that titanium proximal femoral nail is an excellent option for the fixation of inter-trochanteric fractures. The advantages of using this nail are that it is less invasive and prevents excess collapse at the fracture site thus helping to maintain the neck length. It is a load sharing device, hence mobilization can be started as early as the $2^{\text {nd }}$ post operative day. The infection rate is nil and the incidence of mal-union is also less. Thus we conclude that titanium proximal femoral nail when used in the treatment of inter-trochanteric fractures, gives very good functional and radiological outcome. The 
successful outcome of treating inter-trochanteric fractures includes a proper understanding of the biomechanics of the proximal femur, meticulous preoperative planning, careful intra-operative technique and a strict rehabilitation protocol.

\section{Funding: Nil}

Conflict of interest: None.

Permission of IRB: Yes

\section{References}

1. Rang M. Story of Orthopaedics. Philadelphia, Pa: WB Saunders;. 2000:373-395.

2. Kaplan K, Miyamoto R, Levine BR, Egol KA, Zuckerman JD. Surgical management of hip fractures: an evidence-based review of the literature. II: intertrochanteric fractures. J Am Acad Orthop Surg. 2008 Nov. 16(11):665-73.

3. Zuckerman JD, Skovron ML, Koval KJ, Aharonoff G, Frankel VH. Postoperative complications and mortality associated with operative delay in older patients who have a fracture of the hip. J Bone Joint Surg Am. 1995 Oct;77(10):1551-1556.
4. Albareda J, Laderiga A, Palanca D, Paniagua L, Seral F. Complications and technical problems with the gamma nail. Int Orthop. 1996;20(1):47-50.

5. Butt MS, Krikler SJ, Nafie S, Ali MS. Comparison of dynamic hip screw and gamma nail: a prospective, randomised, controlled trial. Injury. 1995;26(9):615618.

6. Boyd HB, Griffin LL: Classification and treatment of trochanteric fractures; Arch Surg 1949; 58(6): 853-866.

7. Kyle RF, Gustilo RB, Premer RF. Analysis of six hundred and twenty-two intertrochanteric hip fractures. J Bone Joint Surg [Am] 1979;61(2):216-21

8. Pajarinen J, Lindahl J, Michelsson O, Savolainen V, Hirvensalo E. Pertrochanteric femoral fractures treated with a dynamic hip screw or a proximal femoral nail: A Randomised study comparing postoperative rehabilitation. J Bone Joint Surg Br. 2005;87-B(1):7681.

9. Morihara T. Proximal femoral nail for treatment of trochanteric femoral fractures.Journal of Orthopaedic Surgery 2007, 15(3):273-7.

\section{How to cite this article?}

Jose Ashish K, Surendher Kumar R. Titanium proximal femoral nailing for inter-trochanteric fractures of femur. Int $J$ Med Res Rev 2015;3(9):990-995. doi: 10.17511/ijmrr.2015.i9.183. 